\title{
Predicting the duration of symptoms in lower respiratory tract infection
}

\author{
Michael Moore, Paul Little, Kate Rumsby, Jo Kelly, Louise Watson, Greg Warner, \\ Tom Fahey and Ian Williamson
}

\author{
ABSTRACT \\ Background \\ Acute lower respiratory tract infection (LRTI) presenting \\ in primary care has a long natural history. Antibiotic \\ treatment makes little or no difference to the duration \\ of cough. Limited information is currently available \\ regarding predictors of illness duration. \\ Aim \\ To determine predictors of illness duration in acute \\ LRTI in primary care. \\ Design of study \\ Secondary analysis of trial data to identify independent \\ predictors of illness severity and duration. \\ Setting \\ Primary care. \\ Method \\ Eight-hundred and seven patients aged 3 years and \\ over with acute illness (21 days or less) presenting with \\ cough as the main symptom plus at least one \\ symptom or sign from sputum, chest pain, dyspnoea, \\ or wheeze were recruited to the study. Main outcomes \\ were duration of symptoms (rated at least a slight \\ problem) and more severe symptoms (rated at least \\ moderately bad). \\ Results \\ The average duration of cough (rated at least a slight \\ problem) was 11.7 days and was shorter among \\ children (duration -1.72 days; $95 \%$ confidence interval \\ $[\mathrm{Cl}]=-3.02$ to -0.41$)$ or in individuals with a history of \\ fever $(-1.22$ days; $95 \% \mathrm{Cl}=-0.18$ to 2.27$)$. The \\ duration of cough was longer among those with \\ restricted activities on the day they saw the doctor \\ (+0.69 days for each point of a 7-point scale). The \\ duration of more severe symptoms was longer in those \\ with a longer duration of symptoms prior to \\ consultation, with a more severe cough on the day of \\ seeing the doctor, and restriction of activities on the \\ day of seeing the doctor.

\section{Conclusion} \\ IIIness duration may be predicted from a limited \\ number of clinical symptoms and from prior history. \\ These findings should be subjected to validation in a \\ separate population. To minimise expectation about \\ rapid resolution of illness, adults who have restricted \\ activities could be advised that they are likely to \\ experience symptoms for longer. \\ Keywords \\ bronchitis; cough; prognosis; respiratory tract \\ infections.
}

\section{INTRODUCTION}

Acute respiratory illness is the most common condition managed in primary care and antibiotics are widely prescribed for lower respiratory tract illness (LRTI) despite limited evidence of benefit. ${ }^{1-3}$ Findings have been reported from a large open pragmatic trial of three different strategies in LRTI (antibiotics, delayed antibiotics, and no antibiotics) and a leaflet among 807 patients with acute LRTI. ${ }^{4}$ No difference was found in illness duration with no offer of antibiotics compared to immediate or delayed antibiotics, and the illness lasted on average almost 12 days following the index consultation.

Reassurance and information for patients presenting with acute respiratory illness has been shown to be a better predictor of satisfaction than the provision of antibiotics, ${ }^{5,6}$ and up to two-thirds of patients may be seeking explanation and reassurance rather than a prescription. ${ }^{7}$ The minority of patients re-consulting with cough within the first month of illness have evidence of persisting infection warranting additional treatment. ${ }^{8}$ Only very limited information regarding the duration of symptoms is currently available in children ${ }^{9-11}$ and adults. ${ }^{12}$ Only one prior study has documented the severity and duration of symptoms and assessed

M Moore, MRCP, FRCGP, senior lecturer; P Little, MSc, PhD, $M R C P, F R C G P$, professor of primary care research; $K$ Rumsby, trial manager; $\boldsymbol{J}$ Kelly, research assistant; $\boldsymbol{L}$ Watson, $M S C$, $P h D$, honorary senior research fellow; I Williamson, FRCGP, senior lecturer, University of Southampton, Division of Community Clinical Sciences; $\mathbf{G}$ Warner, MRCGP, DCG, DRCOG, GP, Nightingale Surgery, Romsey; T Fahey, MD, FRCGP, professor, Department of General Practice, Royal College of Surgeons in Ireland, Dublin, Ireland.

Address for correspondence

Michael Moore, University of Southampton, Division of Community Clinical Sciences, Primary Medical Care Group, Aldermoor Close, Southampton, SO16 5ST.

E-mail: mvm198@soton.ac.uk

Submitted: 28 June 2007; Editor's response: 9 August 2007; final acceptance: 30 October 2007.

(c)British Journal of General Practice 2008; 58: 88-92.

DOI: 10.3399/bjgp08X264045 
predictors of illness duration: a history of asthma predicted prolonged illness whereas fever or perspiring and receipt of antibiotics were associated with a shorter duration. ${ }^{13}$

In this study a secondary analysis of the original trial data ${ }^{4}$ has been undertaken to document the natural history of key symptoms and to determine if any features in the presenting history or examination influence symptom duration following consultation.

\section{METHOD}

The main trial has been fully reported elsewhere. Thirty-six doctors in the Wessex region of the UK recruited 807 ambulant patients. Patients were aged 3 years and over, with acute illness (21 days or less), presenting with cough as the main symptom in primary care, and with at least one symptom or sign localising to the lower tract (sputum, chest pain, dyspnoea, or wheeze)..$^{14}$ The study excluded patients with history or physical examination findings suggestive of pneumonia using the British Thoracic Society guidelines, ${ }^{15}$ that is, new focal chest signs combined with systemic features. Patients were also excluded if they were clinically diagnosed with asthma, other chronic or acute lung diseases, cardiovascular disease, major current psychiatric disorder, mental subnormality or dementia, or if they had complications from previous episodes of LRTI.

Randomisation was undertaken within the consultation to minimally influence natural prescribing behaviour and responses. Patients were randomised to one of six randomised groups defined by two factors.

Factor 1 - leaflet:

- leaflet; or

- no leaflet.

Factor 2 - antibiotic prescription:

- a course of antibiotics;

- no offer of antibiotics; or

- delayed antibiotics - a course of antibiotics on request if symptoms were not resolved after 10 days.

The information leaflet consisted of one page concerning the natural history of LRTI, ${ }^{16,17}$ and addressed the patients' major worries. ${ }^{17}$

\section{Outcome and data collection}

Daily diary and satisfaction questionnaire. Patients, with a parent's help if aged $<16$ years, completed a validated daily symptom diary and recorded their temperature with Tempa•Dot ${ }^{\mathrm{TM}}$ thermometers. ${ }^{18}$ The diary was continued until symptoms settled (maximum 21 days after consultation) with items

\section{How this fits in}

Acute lower respiratory tract infection presenting in primary care has a long natural history. Antibiotic treatment makes little or no difference to the duration of cough, and illness duration may be predicted from the history at

presentation. Adults with a longer prior illness, or who have restricted activities could be advised that they are likely to experience symptoms for longer.

recorded included antipyretic use and six symptoms: cough, dyspnoea, sputum production, wellbeing, sleep disturbance, and activity disturbance. Each was scored $0=$ no problem, $1=$ very little problem, 2 = slight problem, 3 = moderate problem, 4 = bad problem, $5=$ very bad problem, or $6=$ as bad as it could be.

Clinical symptoms and signs. The clinical history and symptoms of patients for example, prior duration of symptoms before the consultation, sputum production, and sputum colour, and signs, for example, breath sounds, either normal or bronchovesicular, or added sounds such as coarse crepitations, were documented initially by the doctor.

\section{Analysis}

The study investigated the influence of presenting history and symptoms on three outcomes using multiple linear regression, retaining variables if they remained significant at the $5 \%$ level. A full list of variables included in the analysis is shown in Tables 1-3. The outcomes were duration of cough until it was a slight problem or less, duration of symptoms until all symptoms were rated as moderately bad or less, and symptom severity scores 2-4 days after seeing the doctor. Since this study was nested within a trial, the multivariate analysis also controlled for the interventions (antibiotic prescribing group and leaflet); however, neither the estimates nor the

Table 1. Diary symptoms duration in days until a slight problem or less (centile distribution).

\begin{tabular}{lccccc} 
Symptom & 10th centile & 25th centile & 50th centile & 75th centile & 90th centile \\
\hline Cough & 5 & 7 & 11 & 17 & 21 \\
\hline Dyspnoea & 0 & 1 & 5 & 10 & 16 \\
\hline Sputum production & 1 & 5 & 8 & 14 & 20 \\
\hline Sleep disturbance & 1 & 4 & 7 & 12 & 16 \\
\hline Activities restriction & 1 & 4 & 7 & 12 & 18 \\
\hline Unwell & 3 & 5 & 8 & 12 & 18 \\
\hline Any symptom & 5 & 7 & 12 & 18 & 21 \\
\hline
\end{tabular}




\begin{tabular}{|c|c|c|c|}
\hline & $\beta(95 \% \mathrm{Cl})$ & Adjusted $\beta^{\mathrm{a}}(95 \% \mathrm{Cl})$ & $P$-value \\
\hline \multicolumn{4}{|l|}{ Clinical assessment } \\
\hline Prior duration, days & 0.10 (0.04 to 0.17$)$ & 0.11 (0.03 to 0.18 ) & $0.004^{b}$ \\
\hline History of fever & $-0.60(-0.36$ to -1.56$)$ & $-1.22(-0.18$ to -2.27$)$ & 0.022 \\
\hline Green-coloured sputum & $-0.19(-1.11$ to 0.73$)$ & $-0.79(-1.79$ to 0.21$)$ & 0.119 \\
\hline Sore throat & $0.61(-0.38$ to 1.59$)$ & $0.21(-0.86$ to 1.27$)$ & 0.702 \\
\hline Runny nose & $-1.43(-0.43$ to -2.42$)$ & $-1.35(-0.31$ to -2.39$)$ & 0.011 \\
\hline Prolonged expiration & 3.08 (0.22 to 5.94$)$ & 1.06 (4.23 to -2.11$)$ & 0.512 \\
\hline Respiration rate, $\geq 20 / \mathrm{min}$ & $-0.06(-1.14$ to 1.02$)$ & $0.04(-0.06$ to 0.15$)$ & 0.415 \\
\hline Temperature, $\geq 37.0^{\circ} \mathrm{C}$ & $-1.15(-2.15$ to -0.15$)$ & -1.05 (0.03 to -2.12$)$ & 0.056 \\
\hline Coarse crepitations & $0.58(-0.72$ to 1.89$)$ & $0.09(-1.28$ to 1.45$)$ & 0.901 \\
\hline Wheeze & $0.69(-0.71$ to 2.08$)$ & $0.17(-1.32$ to 1.70$)$ & 0.807 \\
\hline Fine crepitations & $-0.56(-3.41$ to 2.29$)$ & $0.02(-2.94$ to 2.97$)$ & 0.991 \\
\hline Smoker & $-0.03(-1.31$ to 1.26$)$ & $-0.19(-1.62$ to 1.24$)$ & 0.795 \\
\hline \multicolumn{4}{|l|}{ Age } \\
\hline Older age, $\geq 60$ years & $-0.99(-3.08$ to 0.22$)$ & $-0.94(-2.22$ to 0.34$)$ & 0.149 \\
\hline Children, $<16$ years & $-1.87(-3.08$ to -0.65$)$ & $-1.72(-3.02$ to -0.41$)$ & $0.010^{\mathrm{b}}$ \\
\hline \multicolumn{4}{|l|}{ Symptoms on day 1} \\
\hline Cough & $0.62(0.19$ to 1.06$)$ & $0.36(-0.12$ to 0.84$)$ & 0.137 \\
\hline Sputum & 0.34 (0.02 to 0.67$)$ & $0.14(-0.20$ to 0.49$)$ & 0.42 \\
\hline Short of breath & $0.22(-0.10$ to 0.55$)$ & $-0.03(-0.39$ to 0.32$)$ & 0.851 \\
\hline Sleep & $0.28(-0.02$ to 0.58$)$ & $0.00(-0.34$ to 0.35$)$ & 0.986 \\
\hline Restriction of activities & 0.57 (0.25 to 0.89$)$ & 0.69 (0.36 to 1.02$)$ & $<0.001^{\mathrm{b}}$ \\
\hline Unwell & 0.35 (0.01 to 0.69$)$ & $-0.03(-0.53$ to 0.47$)$ & 0.909 \\
\hline
\end{tabular}

The $\beta$ estimates are the estimate of the number of days' difference in duration attributable to each variable. ${ }^{\text {aT }}$ The estimates for each variable controls for the effects of the other variables and the randomised interventions. ${ }^{\mathrm{b}} \mathrm{P}$-value is significant at $\leq 0.01$.

\section{Table 3. Predictors of duration of moderately bad symptoms.}

\begin{tabular}{lcccc} 
& $\beta(95 \% \mathrm{Cl})$ & Adjusted $\beta^{\mathrm{a}}$ (95\% Cl) & $P$-value \\
\hline Clinical assessment & & & \\
$\quad$ Prior duration, days & $0.07(0.01$ to 0.13$)$ & $0.10(0.04$ to 0.15$)$ & $0.001^{\mathrm{b}}$ \\
History of fever & $0.51(-0.34$ to 1.36$)$ & $-0.03(-0.86$ to 0.80$)$ & 0.95 \\
Green-coloured sputum & $0.26(-0.56$ to 1.07$)$ & $0.16(-0.61$ to 0.94$)$ & 0.68 \\
Sore throat & $-0.03(-0.91$ to 0.84$)$ & $-0.14(-0.97$ to 0.69$)$ & 0.74 \\
Runny nose & $-0.55(-1.42$ to 0.33$)$ & $-0.61(-1.42$ to 0.20$)$ & 0.14 \\
Prolonged expiration & $1.32(-1.41$ to 4.05$)$ & $0.73(-1.79$ to 3.25$)$ & 0.57 \\
Respiration rate, $\geq 20 /$ min & $-0 . .08(-1.05$ to 0.88$)$ & $0.02(-1.05$ to 0.26$)$ & 0.97 \\
Temperature, $\geq 37.0^{\circ} \mathrm{C}$ & $-0.68(-1.55$ to 0.20$)$ & $-0.86(-1.68$ to -0.04$)$ & 0.04 \\
Coarse crepitations & $-0.03(-1.17$ to 1.10$)$ & $-0.02(-1.09$ to -1.04$)$ & 0.96 \\
Wheeze & $-0.07(-1.32$ to 1.17$)$ & $-0.14(-1.31$ to 1.03$)$ & 0.82 \\
Fine crepitations & $-0.87(-3.32$ to 1.58$)$ & $-0.18(-2.51$ to 2.16$)$ & 0.88 \\
Smoker & $-0.13(-1.29$ to 1.04$)$ & $-0.08(-1.19$ to 1.02$)$ & 0.88 \\
\hline Age & & & \\
Older age, $\geq 60$ years & $-1.52(-2.59$ to 0.45$)$ & $-0.81(-1.83$ to 0.20$)$ & 0.12 \\
Children, $<16$ years & $-0.72(-1.82$ to 0.37$)$ & $-0.20(-1.25$ to 0.85$)$ & 0.71 \\
\hline Symptoms on day 1 & & & \\
Cough & $1.04(0.69$ to 1.39$)$ & $0.68(0.30$ to 1.05$)$ & $<0.001^{\text {b }}$ \\
Sputum & $0.58(0.32$ to 0.85$)$ & $0.21(-0.07$ to 0.48$)$ & 0.14 \\
Short of breath & $0.52(0.25$ to 0.78$)$ & $0.06(-0.23$ to 0.34$)$ & 0.69 \\
Sleep & $0.63(0.38$ to 0.89$)$ & $0.11(-0.17$ to 0.40$)$ & 0.43 \\
Restriction of activities & $0.91(0.65$ to 1.17$)$ & $0.82(0.54$ to 1.11$)$ & $<0.001^{b}$ \\
Unwell & $0.89(0.61$ to 1.16$)$ & $0.21(-0.20$ to 0.63$)$ & 0.31 \\
\hline
\end{tabular}

The $\beta$ estimates are the estimate of the number of days' difference in duration attributable to each variable. ${ }^{a}$ The estimates for each variable controls for the effects of the other variables and the randomised interventions. ${ }^{\circ} \mathrm{P}$-value is significant at $\leq 0.01$. inferences were altered by inclusion of the randomisation groups. Based on the data in upper respiratory tract infection (URTI), it was hypothesised that those with fever/temperature would settle more quickly; it was also hypothesised that the severity of symptoms on the day of consultation would predict symptom severity and duration.

\section{RESULTS}

The study sample of 807 patients included 136 children (aged <16 years) and 133 older patients (aged $>60$ years). Completed symptom diaries were returned by $562(70 \%)$ patients and an additional 78 (10\%) patients provided information about symptom duration and severity in a telephone interview. A previous study showed no effect of either the information leaflet or withholding or delaying antibiotics on the main outcomes. ${ }^{4}$ Twenty-five per cent of patients rated their cough as at least a slight problem for 17 days or more, and $25 \%$ had most other symptoms for about 2 weeks (Table 1). The cough (until a slight problem or less) lasted on average 11.7 days following the index consultation. To reduce the chance of type 1 error, comments on the results of the multivariate analysis are restricted to those achieving a significance of 0.01 or less.

\section{Predictors of cough duration}

The duration of cough was approximately 1 day shorter in children. It was longer in those with a longer prior history and among those with restricted activities on the day that they saw the doctor (+0.69 days for each point on a 7-point scale) (Table 2).

\section{Predictors of numbers of days with moderately bad symptoms}

The duration of moderately bad symptoms is defined as the number of days high-scoring symptoms were recorded (score entries of $\geq 4$ ). Those with a longer prior duration of illness, with more severe cough on presentation, and restriction of activities all suffered from a longer duration of more severe illness (Table 3). Following the index consultation, the subsequent duration of moderately bad symptoms was found to be 0.1 days longer for every day of prior illness (95\% confidence interval $[\mathrm{Cl}]=0.04$ to 0.15 ), and 0.68 days longer for those with a more severe cough (that is, 1 scale point worse on a self-rated scale of 7 points of cough severity) $(95 \% \mathrm{Cl}=0.30$ to 1.05$)$. Where none of these variables apply the expected duration of moderately bad symptoms is only 3 days, but when all three apply (prior duration > 10 days; cough a moderately bad problem; and restriction of activities a moderately bad problem) the duration of moderately bad symptoms is predicted to be 9 days. 


\section{Predictors of symptom severity for days 2-4 after seeing the doctor}

Children and adults aged $\geq 60$ years were found to be less unwell 2-4 days after the index consultation. Those with more severe symptoms other than cough on presentation were more likely to remain unwell after 2-4 days. No other factors in the history or examination influenced the severity of symptoms after 2-4 days, which is dependent predominantly on symptom severity at presentation (Table 4).

\section{DISCUSSION}

\section{Summary of main findings}

This study has provided detailed natural history information for the symptoms that most concern patients with LRTI. IIIness duration may be predicted according to the severity of symptoms, but few variables from clinical examination are important. To minimise expectation about rapid resolution of illness, adults with a longer prior illness or who have restricted activities can be advised that they are likely to experience symptoms for longer.

At the start of the study it was hypothesised that those with fever would have a shorter duration of illness. The results provide some support for this hypothesis in that those with a history of fever had a shorter duration of cough until experiencing a slight problem or less $(P=0.022)$, and those with a temperature of $37.0^{\circ} \mathrm{C}$ or higher were also more likely to have shorter duration $(P=0.056)$. Those with a recorded fever also had a shorter duration of moderately bad symptoms $(P=0.04)$ although none of these results achieved the level of significance set for this analysis.

A biological explanation for this finding might be that those patients who exhibit a brisk immune response, characterised by raised temperature, are more likely to get better sooner. These findings are consistent with previous findings from studies of patients with URTI ${ }^{18}$ and $\mathrm{LRTI}^{13}$ but require further replication in a different population of patients.

\section{Strengths and limitations of the study}

This study of 807 patients with LRTI presenting in primary care is the largest to date in the disease area, and the only study to assess the predictors of illness duration in adults. The physical examination was recorded on a structured datasheet and all information was prospectively gathered. The use of a daily symptom diary by patients allowed detailed information to be captured regarding LRTI natural history, which included the severity of a range of symptoms that patients had previously reported to be of concern. ${ }^{14}$ The Likert scales previously have been shown to be reliable, to have good construct validity, predict illness duration, ${ }^{19}$ and the diary has been
Table 4. Predictors of symptom severity for days 2-4 after seeing the doctor.

\begin{tabular}{lccc} 
& $\beta(95 \% \mathrm{Cl})$ & Adjusted $\beta^{\mathrm{a}}(95 \% \mathrm{Cl})$ & $P$-value \\
\hline Clinical assessment & & & \\
$\quad$ Prior duration, days & $0.00(-0.01$ to 0.01$)$ & $0.00(-0.005$ to 0.01$)$ & 0.34 \\
History of fever & $0.44(0.26$ to 0.62$)$ & $0.06(-0.08$ to 0.19$)$ & 0.42 \\
Green-coloured sputum & $0.12(-0.06$ to 0.29$)$ & $-0.04(-0.17$ to 0.10$)$ & 0.34 \\
Sore throat & $0.11(-0.08$ to 0.30$)$ & $0.01(-0.13$ to 0.15$)$ & 0.86 \\
Runny nose & $0.06(-0.12$ to 0.25$)$ & $-0.06(-0.19$ to 0.08$)$ & 0.43 \\
Prolonged expiration & $0.31(-0.21$ to 0.84$)$ & $0.04(-0.39$ to 0.46$)$ & 0.87 \\
Respiration rate, $\geq 20 /$ min & $0.07(-0.14$ to 0.27$)$ & $-0.04(-0.20$ to 0.11$)$ & 0.60 \\
Temperature, $\geq 37.0^{\circ} \mathrm{C}$ & $-0.06(-0.25$ to 0.13$)$ & $-0.07(-0.21$ to 0.07$)$ & 0.33 \\
Coarse crepitations & $0.09(-0.16$ to 0.33$)$ & $0.02(-0.16$ to 0.20$)$ & 0.83 \\
Wheeze & $0.18(-0.09$ to 0.44$)$ & $-0.04(-0.23$ to 0.16$)$ & 0.70 \\
Fine crepitations & $-0.04(-0.57$ to 0.49$)$ & $0.16(-0.22$ to 0.54$)$ & 0.42 \\
Smoker & $0.33(0.10$ to 0.56$)$ & $0.02(-0.16$ to 0.20$)$ & 0.80 \\
\hline Age & & & \\
Older age, $\geq 60$ years & $-0.53(-0.31$ to 0.75$)$ & $-0.17(-0.01$ to -0.34$)$ & 0.043 \\
Children, $<16$ years & $-0.58(-0.35$ to -0.80$)$ & $-0.23(-0.06 ;$ to -0.41$)$ & $0.008^{\mathrm{b}}$ \\
\hline Symptoms on day 1 & & & \\
Cough & $0.36(0.29$ to 0.43$)$ & $0.03(-0.03$ to 0.10$)$ & 0.33 \\
Sputum & $0.30(0.25$ to 0.35$)$ & $0.15(0.10$ to 0.19$)$ & $<0.001^{\mathrm{b}}$ \\
Short of breath & $0.31(0.25$ to 0.36$)$ & $0.12(0.07$ to 0.17$)$ & $<0.001^{\mathrm{b}}$ \\
Sleep & $0.28(0.24$ to 0.33$)$ & $0.11(0.07$ to 0.16$)$ & $<0.001^{\mathrm{b}}$ \\
Restriction of activities & $0.40(0.35$ to 0.45$)$ & $0.17(0.11$ to 0.24$)$ & $<0.001^{\mathrm{b}}$ \\
Unwell & $0.40(0.34$ to 0.45$)$ & $0.10(0.03$ to 0.17$)$ & $0.003^{\mathrm{b}}$ \\
\hline
\end{tabular}

The $\beta$ estimates are the estimate of the number of days' difference in duration attributable to each variable. ${ }^{a}$ The estimates for each variable controls for the effects of the other variables and the randomised interventions. ${ }^{b} \mathrm{P}$-value is significant at $\leq 0.01$.

validated. ${ }^{18}$ Since clinical findings are part of routine practice, no special training was provided to practitioners. Examination findings are therefore not standardised but variation is likely to mimic that found in routine practice. Secondary analysis must always be treated with some caution and should ideally be subject to confirmation in an independent data set.

The analysis revealed some predictors of illness duration, which together have moderate predictive value for total symptom duration. In this UK population, patients delay a considerable time prior to consultation. This may reflect both access and cultural factors, and so may be different in other countries. Equal caution should be exercised when generalising outside the setting of the original study.

\section{Comparison with existing literature}

Patients presenting with LRTI in this study had symptoms for 9 days prior to consultation and were, on average, likely to continue to suffer a cough rated as at least a slight problem for the next 12 days, with a total likely illness duration of 3 weeks. ${ }^{4}$ The data are in concordance with limited previous prospective cohort data from patients presenting with LRTI in whom physical signs were documented, ${ }^{13}$ and from diary information completed 10 days after consultation where $58 \%$ of patients were still 
coughing. ${ }^{12}$ More detailed studies in preschool children using a similar diary card to the current study have described similarly protracted illness and illustrated the difficulty of predicting those individuals expected to have a longer duration..$^{9-11}$

\section{Implications for clinical practice and future research}

Patients have different expectations from doctors regarding respiratory illness and the need for antibiotics, ${ }^{20}$ and those who consult are more worried by their symptoms and consider their symptoms to be severe and interfere with social roles. ${ }^{16}$ It is important to address these worries and expectations since doctors overestimate the pressure to prescribe antibiotics in a variety of situations. ${ }^{5}$ This study provides further information for practitioners to aid these discussions in routine consultations for one of the commonest acute conditions managed in primary care.

It may be reassuring for parents that their children are likely to experience a shorter illness than adults. Restriction of normal daily activities is a useful indication of the severity of the illness, and such patients can also be warned that resolution is unlikely to be quick. These prognostic data should help practitioners in their discussions in routine consultations, so maximising realistic expectations, increasing satisfaction, and providing reassurance that most coughs will last on average for 3 weeks, irrespective of treatment.

\section{Funding body}

This work was funded by the Medical Research Council (G108/322[2])

\section{Ethical approval}

This study was approved by the South West Multi Research Ethics Committee (MREC/99/6/35)

\section{Competing interests}

The authors have stated that there are none Acknowledgements

We are very grateful to the patients and practices who agreed to participate

\section{Discuss this article}

Contribute and read comments about this article on the

Discussion Forum: http://www.rcgp.org.uk/bjgp-discuss

\section{REFERENCES}

1. Huchon GJ, Gialdroni-Grassi G, Leophonte P, Manresa F. Initial antibiotic therapy for lower respiratory tract infection in the community: a European Survey. Eur Resp J 1996; 9: 1590-1595.

2. HMSO, OPCS. Morbidity statistics from general practice: fourth national study 1991. London: HMSO, 1994.

3. Smucny JJ, Becker LA, Glazier RH, McIsaac W. Are antibiotics effective treatment for acute bronchitis? A meta-analysis. J Fam Pract 1998; 47(6): 453-460.

4. Little P, Rumsby K, Kelly J, et al. Information leaflet and antibiotic prescribing strategies for acute lower respiratory tract infection: a randomized controlled trial. JAMA 2005; 293(24): 3029-3035.

5. Welschen I, Kuyvenhoven M, Hoes A, Verheij T. Antibiotics for acute respiratory tract symptoms: patients' expectations, GPs' management and patient satisfaction. Fam Pract 2004; 21(3): 234-237.

6. Van Duijn HJ, Kuyvenhoven M, Schellevis F, Verheij T. Illness behaviour and antibiotic prescription in patients with respiratory tract symptoms. Br J Gen Pract 2007; 57(540): 561-568.

7. Butler CC, Rollnick S, Pill R, et al. Understanding the culture of prescribing: qualitative study of general practitioners' and patients' perceptions of antibiotics for sore throats. BMJ 1998; 317(7159): 637-642.

8. Macfarlane J, Prewett J, Rose D, et al. Prospective case-control study of role of infection in patients who reconsult after initial antibiotic treatment for lower respiratory tract infection in primary care. $B M$ J 1997; 315(7117): 1206-1210.

9. Butler CC, Hood K, Kinnersley P, et al. Predicting the clinical course of suspected acute viral upper respiratory tract infection in children. Fam Pract 2005; 22(1): 92-95.

10. Hay AD, Wilson A, Fahey T, Peters TJ. The duration of acute cough in pre-school children presenting to primary care: a prospective cohort study. Fam Pract 2003; 20(6): 696-705.

11. Hay $\mathrm{AD}$, Wilson $\mathrm{AD}$. The natural history of acute cough in children aged 0 to 4 years in primary care: a systematic review. $\mathrm{Br} \mathrm{J} \mathrm{Gen}$ Pract 2002; 52(478): 401-409.

12. Holmes WF, Macfarlane JT, Macfarlane RM, Hubbard R. Symptoms, signs, and prescribing for acute lower respiratory tract illness. Br J Gen Pract 2001; 51(464): 177-181.

13. Hopstaken RM, Coenen S, Butler CC, et al. Prognostic factors and clinical outcome in acute lower respiratory tract infections: a prospective study in general practice. Fam Pract 2006; 23(5): 512-519.

14. Macfarlane J, Holmes W, Gard P, et al. Prospective study of the incidence, aetiology and outcome of adult lower respiratory tract illness in the community. Thorax 2001; 56(2): 109-114.

15. British Thoracic Society Guidelines for the Management of Community Acquired Pneumonia. Thorax 2001; 56(suppl IV): $1-64$.

16. Macfarlane JT, Holmes WF, Macfarlane RM, Lewis S. Reducing reconsultation for acute lower respiratory tract illness with an information leaflet: a randomised controlled study of patients in primary care. Br J Gen Pract 1997; 47: 719-722.

17. Cornford CS. Why patients consult when they cough: a comparison of consulting and non-consulting patients. Br J Gen Pract 1998; 48: $1751-1754$.

18. Watson L, Little P, Moore M, et al. Validation study of a diary for use in acute lower respiratory tract infection. Fam Pract 2001; 18(5): 553-554.

19. Little P, Williamson I, Warner G, et al. Open randomised trial of prescribing strategies in managing sore throat. BMJ 1997; 314(7082): 722-727.

20. Van DH, Kuyvenhoven M, Welschen I, et al. Patients' and doctors' views on respiratory tract symptoms. Scand J Prim Health Care 2002; 20(4): 201-202. 